\title{
PAST FLUORIDE EXPOSURE FROM INGESTED TOOTHPASTE EXTRAPOLATED FROM YOUNGER SIBLINGS AND FLUOROSIS STATUS
}

B.S. Tan, I.A. Razak, L.C. Foo. Past fluoride exposure from ingested toothpaste extrapolated from younger siblings and fluorosis status. Annal Dent Univ Malaya 2004; 11: 30-36.

\begin{abstract}
The aim of the study was to assess the association between past fluoride exposures from ingested toothpaste and current fluorosis manifestations in 1011 year old index subjects. Fluorosis was assessed with the Dean's Index in 1343 10-11 year old index subjects. Two hundred index subjects who had younger 4-5 year old siblings were sub sampled and fluoride exposures from ingested toothpaste in their younger siblings (proxy subjects) were determined. The values for the fluoride ingested per brushing of the proxy subjects were utilised together with past frequency of toothbrushing of index subjects to extrapolate on the past exposure of index subjects.

The mean extrapolated past fluoride exposure from ingestion of toothpaste was highly variable; $671.7 \mathrm{ug} \pm 739.3 \mathrm{ug}(\mathrm{sem}=56.9)$. It was higher in the subjects with fluorosis $(697.3 \mathrm{ug}$ ) than in those without fluorosis $(646.89 \mathrm{ug})$ but differences were not of statistical significance. This approach of extrapolation has not been reported elsewhere and need to be validated. The implications of the present methodology to estimate past fluoride exposure is discussed.
\end{abstract}

Key words: fluoride exposure, ingested dentifrice, Malaysia.

\section{INTRODUCTION}

Fluorosis is a condition whereby opacities ranging from thin white opaque lines to coalesced blotchy cloudy areas occur in the enamel as a result of exposures to fluoride during enamel development. The greater the fluoride intake, the more severe are the clinical manifestations of fluorosis $(1,2)$. Fluoride intake can come from food, drinking water, beverages and fluoride containing dental and pharmaceutical products.

In the late 1990's there was a spate of antifluoride sentiments in Malaysia. Consumer groups expressed concerns of possible excessive fluoride exposure of individuals, especially children, including that which may result from ingestion of fluoridated toothpaste.

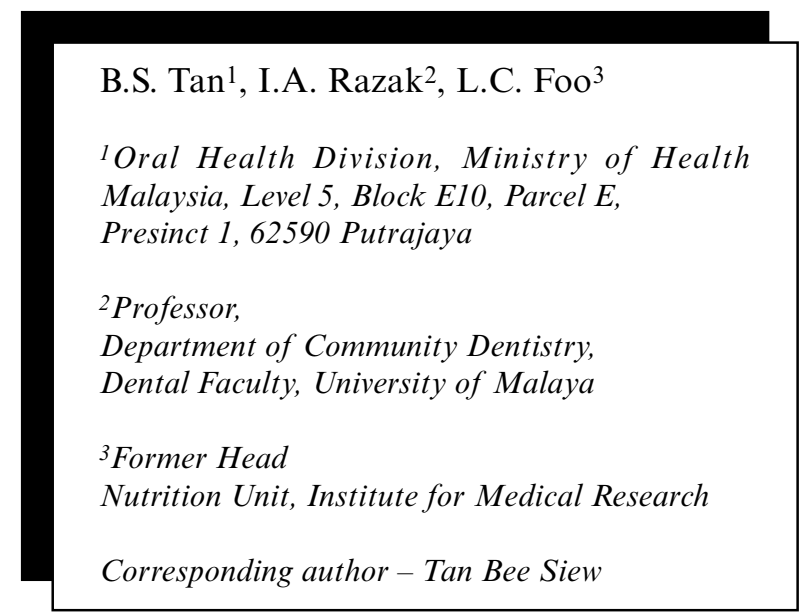

Fluoridated toothpaste accounts for more than $90 \%$ of the US (3) and Canadian (4) market. An estimated $97.0 \%$ of the toothpaste in Denmark contains fluoride (5) and over $99.4 \%$ in the Malaysia market was reported to consist of fluoridated toothpaste (6).

Fluoride from toothpaste has been shown to be readily bioavailable $(7,8)$. The ready bioavailability of fluoride from toothpaste, implies that fluoride toothpaste may play an important role in daily exposure of children to fluoride. Murakami et al., (9) found $66.7,70.0$ and $85.3 \%$ use of fluoridated toothpaste.in 3, 4 and 5 year olds respectively. The mean daily fluoride toothpaste (in $\mathrm{g}$ ) used by these children were $0.392 \pm 0.221,0.360 \pm 0.258,0.464$ \pm 0.215 g respectively.

Studies on use of fluoridated toothpaste are numerous. Many were on the amount of toothpaste used per se $(6,10-13)$, others were on fluoride ingestion per se $(5,14-17)$ with no attempts to relate to fluorosis. No local studies have ever been conducted to determine fluoride toothpaste ingestion in Malaysian children at the time of their enamel formation.

\section{Objectives}

The aim of the study was to relate past fluoride exposures (at the time of enamel formation) from ingested toothpaste to current fluorosis manifestations in 10-11 year old index subjects. The objective of this study were to:

i) determine fluorosis status of the 10-11 year old index subjects,

ii) determine the fluoride exposures from toothpaste ingestion in their 4-5 year old siblings, 
iii) estimate past intake of fluoride from toothpaste ingested at the time of enamel formation of index subjects by extrapolating from (ii) above and then

iv) relating this past exposure (iii) to fluorosis manifestation (i).

\section{MATERIALS AND METHODS}

Fluorosis is seen in the permanent dentition (and rarely in the deciduous teeth) whilst exposure to fluoride would have occurred during enamel formation in the earlier years. To overcome the problem of a longitudinal study required due to the long natural history of fluorosis, an analytical crosssectional design was used. Disease (fluorosis in index subjects) was measured simultaneously with exposures (in the younger siblings i.e. proxy subjects) to assess the association between the past exposures and existing disease. The picture of past exposure, ideally thus, need be captured in proxies from the ages 4-5 months to 7 years. However, as this investigation was part of a larger study where total fluoride intake was determined from urinary fluoride excretions, the need for 24-hour urine collections, imposed a limitation to the age of the younger siblings. 24-hour urine collection in individuals of 3 years and below is difficult, inaccurate and unreliable due to poor compliance and consequently impractical. Thus the study was limited to 4-5 year old siblings.

The study was conducted in Selangor, a fluoridated area centrally located on the west coast of peninsular Malaysia. Fluorosis was assessed in 10-11 year old Index subjects using the Dean's Index $(18,19)$. The $4-5$ year old younger siblings (if any) of these subjects were then used as proxies for past fluoride exposures.

A questionnaire (QA) survey was conducted to elicit socio-demographic details and past oral hygiene practices of the 10-11 year old Index subjects, and also the existing oral hygiene $(\mathrm{OH})$ practices of the Proxy subjects where applicable. These two questionnaires were brought home and administered by the parents.

Ingestion of toothpaste was determined by the method of difference between the amount taken for use less the amount used but not ingested using a duplicate technique. Packets of pairs of identical toothbrushes were given to the subjects. Each time that he/she brushed, the subject was instructed to dispense the amount of toothpaste, as would be normally used, onto both the toothbrushes in the pair. One toothbrush was placed back into the packet labelled "NOT USED" with the dispensed toothpaste and kept for collection and subsequent measurement. The other toothbrush was then used. Upon finishing brushing, the used toothbrush with residual toothpaste, was not to be washed/rinsed, but was placed into a separate plastic bag labelled "USED" and kept for subsequent collection. Instructions were also given to the child to expell his rinses into plastic receptacles. Enough sample toothbrushes and receptacles were given to the child for the duration of the study. Specimens of toothbrushes (used and not used) and rinses were collected over the same two days. Enough toothbrushes and receptacles were given to the child for the 2-day study.

All fluoride determinations were made with the selective ion meter in conjunction with a fluoride ion electrode. From the determinations of fluoride in the dispensed toothpaste, the residual toothpaste, the rinses and the specimens of water used for rinsing, the amount of fluoride from ingested toothpaste was determined:

$$
F_{I}=F_{D}-F_{R}-\left(F_{\text {EXP }}-F_{\text {RINSE WATER }}\right) \text {, where }
$$

$\mathrm{F}_{\mathrm{I}}=$ Fluoride from ingested toothpaste,

$\mathrm{F}_{\mathrm{D}}=$ Fluoride in toothpaste dispensed,

$\mathrm{F}_{\mathrm{R}}=$ Fluoride in residual toothpaste,

$\mathrm{F}_{\text {EXP }}=$ Fluoride in expectorant,

$\mathrm{F}_{\text {RINSE WATER }}=$ Fluoride in water used for rinsing

Using these values of fluoride from ingested toothpaste, the amounts of fluoride ingested per brushing were then obtained. These latter values were then utilised together with the past frequency of brushing of index subjects to extrapolate on the amounts of fluoride that would have been ingested from swallowed toothpaste by the index subjects, $F_{I}$ (index), in the past.

Data were analysed using the SPSSPC+ statistical package. The t-test and ANOVA were used to compare differences in means of variables between two and more groups respectively. The Levene's test was used to determine homogeneity of variances, and the $p$ values corresponding to the assumed equality or non-equality of the variances were then determined. Level of significance was set at $\alpha=0.05$.

\section{RESULTS}

A total of 1343 Index subjects were examined for fluorosis using the Dean's Index as part of a larger study. Of these, 342 subjects had 4-5 year old siblings. From these subjects with 4-5 year old siblings, 200 subjects were sub sampled. This paper focuses on relating the fluorosis seen to fluoride exposure from ingested toothpaste extrapolated for the past of this sub sample.

Specimens of rinses were returned from 182 subjects, specimens of dispensed toothpaste and residual toothpaste were obtained from 180 subjects, 174 subjects returned both toothpaste samples and rinses. Of these, however, owing to either differences between dispensed amounts and residual amounts being smaller than the amount of fluoride 
Table 1. Fluoride from ingested toothpaste - proxy subjects

\begin{tabular}{lrrrr}
\hline & Mean & Median & SD & SEM \\
\hline Toothpaste & & & & \\
F in toothpaste dispensed (ug) & 1296.6 & 1057.7 & 974.8 & 72.6 \\
F in residual toothpaste (ug) & 444.8 & 315.0 & 399.8 & 29.8 \\
Rinses & & & & \\
Rinse volumes (ml) & 204.6 & 140.5 & 225.5 & 16.7 \\
Rinse [F] (ppm) & 6.3 & 2.90 & 1.12 & 0.8 \\
F in rinses (ug) & 728.3 & 472.3 & 1444.5 & 107.1 \\
F rinse water & 114.3 & 70.4 & 143.2 & \\
F expectorated (corrected for F from water used) & 499.7 & 368.0 & 520.7 & 22.0 \\
\hline F I (proxy) /2 days & 426.9 & 279.6 & 505.5 & 38.9 \\
\hline
\end{tabular}

Table 2. Frequency of brushing of subjects

\begin{tabular}{lcrr}
\hline Subjects & $\begin{array}{c}\text { Freq of brushing/ } \\
48 \text { hours }\end{array}$ & $\mathrm{n}$ & $\%$ \\
\hline \multirow{2}{*}{ Proxy } & 1 & 9 & 5.4 \\
& 2 & 41 & 24.6 \\
& 3 & 28 & 16.8 \\
& 4 & 87 & 52.1 \\
& 6 & 2 & 1.2 \\
\hline Total & 167 & 100 \\
\hline Index & & & \\
\hline & Past frequency of & $\mathrm{n}$ & $\%$ \\
brushing/day & & \\
\hline & 0 & 6 & 3.6 \\
& 1 & 74 & 44.3 \\
& 2 & 61 & 36.5 \\
& 3 & 22 & 13.2 \\
4 & 4 & 2.4 \\
\hline & Total & 167 & 100 \\
\hline
\end{tabular}

expectorated into rinses, or the dispensed amounts being smaller than residual amounts, only 167 specimens were analysable for the amount of fluoride from ingested toothpaste.

Tables 1 and 2 respectively refer to the values of the parameters relevant for the computation of the amount of fluoride from ingested toothpaste in the proxy subjects, and the frequencies of brushing of proxy as well as index subjects. Using the values of fluoride from ingested toothpaste in the proxy subjects, the amounts of fluoride ingested per brushing were then obtained. These latter values were then utilised together with the past frequency of brushing of index subjects to extrapolate on the amounts of fluoride that would have been ingested from swallowed toothpaste by the index subjects, $F_{I}$ (index), in the past (Table 3 )
Table 3. Fluoride from ingested toothpaste (extrapolated for index subjects' past)

\begin{tabular}{lcrrr}
\hline Parameter & Mean & Median & SD & SEM \\
\hline F I (proxy) per brushing & 131.9 & 84.8 & 151.7 & 11.7 \\
F I (index) / 2 days & 671.7 & 424.5 & 739.3 & 56.9 \\
\hline
\end{tabular}

The mean extrapolated past fluoride exposure from ingestion of toothpaste over the 2 study days was highly variable; $671.7 \mathrm{ug} \pm 739.3 \mathrm{ug}$ $(\mathrm{sem}=56.9)$. It was higher compared to the proxy subjects' mean current exposure over the 2 study days $426.9 \mathrm{ug} \pm 505.5 \mathrm{ug}(\mathrm{sem}=38.9)$. It was higher in the index subjects with fluorosis $(697.3 \mathrm{ug})$ than in those without fluorosis $(646.9 \mathrm{ug})$ but differences were not of statistical significance.

Subjects who started brushing their teeth earlier i.e. at 2 years or less did not show significant increased risk of fluorosis $(79.8 \%$ versus $70.6 \%$; $\mathrm{p}=0.9)$ nor increased ingestion of fluoride from toothpaste $(732.1 \mathrm{ug}$, sem 92.9 versus $590.3 \mathrm{ug}$, sem 72.6, $\mathrm{p}=0.2$ ). Early start to toothpaste usage (at 2 years or less) also did not pose significant increased risk of fluorosis $(43 \%$ versus $57 \% ; p=0.4)$ nor increased toothpaste ingestion (780.4 ug, sem 104.6 versus $592.3 \mathrm{ug}$, sem $68.2 ; \mathrm{p}=0.1)$ as compared to subjects who started toothpaste usage later.

\section{DISCUSSION}

To overcome the problem of a longitudinal study required due to the long natural history of fluorosis, the analytical cross-sectional design was used; disease (in index subjects) being measured simultaneously with exposures (in the siblings i.e. proxy subjects) to assess the association between the exposure variables and existing disease. As had been 
detailed in the previous section, specimens to measure fluoride exposure had all been obtained from the subjects' 4-5 year old sibling. The results were then used to extrapolate on the past exposures of the index subjects taking cognisance of any changes, in the frequencies of brushing which might have affected these values. It was assumed that from the critical period of tooth development of the index subjects to the study period that:

A) oral hygiene practices were similar for each sibling pair, being subjected to the same parental influences, and

B) biological assimilations, susceptibilities and responses to fluoride in each sibling pair were similar.

An internally valid study is one in which the measure of effect obtained in the study agrees with the measure of effect that truly exists in the study population) (16). In this study, any major shifts in any of the above assumptions would lead to problems of internal validity and mask the true fluoride exposurefluorosis relationship. The findings of the present study were therefore interpreted and discussed within these limitations.

The discussion that follows was based on the extrapolated values.

The findings of several studies on ingestion of toothpaste are tabulated in Table 4 . In the present study, the proportion of dispensed toothpaste that was ingested in the proxy subjects was $32.9 \%$ (Table 1 : $\mathrm{F}_{\mathrm{I}}$ (proxy)/2days divided by $\mathrm{F}$ in toothpaste dispensed; i.e. $426.9 \div 1296.6=32.9$ ). It was assumed to be the same for the index subjects. This was comparable to that of 5-year olds reported in Canada (3), the 2-4 year olds in Barnhart's study (15), the 35 year olds in Quebec (21), Ericson and Forsman's (22) 4-5 year old subjects and those of Hargreaves (14) and Salama et al (23).

Absolute quantities of fluoride ingested in this study were however lower than those of children in the studies of Simard et al., (3), Naccache et al., (24) and Rojas-Sanchez (25). The differences between results of the present study with that of Simard and co-workers (3) were probably due to differences in supervision during tooth brushing (nearly all children brushed their teeth by themselves as opposed to $81.7 \%$ being supervised in this study). The subjects of the study of Simard et al, (3) also reported higher frequencies of brushing $(71.4 \%$ brushed twice, $23.8 \%$ thrice and only $4.8 \%$ once, as compared to $36.5 \%$, $13.2 \%$ and $44.3 \%$ respectively in this study). There were also differences in rinsing habits $(78 \%$ as compared to $97.9 \%$ ) in the study of Simard et al., and in the present study, respectively. The subjects in Naccache's study (21) had used $0.24 \%$ sodium fluoride (1000 ppm F) paste whereas in the present study $62.4 \%$ used normal fluoride formulation toothpaste and $37.6 \%$ had used low fluoride formulations (as ascertained from subjects own description of toothpaste used). The study by RojasSanchez et al., (25) had been on a largely younger cohort and ingestion of toothpaste is known to increase with decreasing age.

The amount of fluoride ingested per day in this study was higher than that reported by Villa et al., (26) in Chile. This was possibly because in the Chilean study a standard "kiddies" toothpaste with $500 \mathrm{ppm}$ fluoride was used. Also the amount used in Chile could be artificial; being small amounts that were weighed by research assistants.

The present findings were also higher that those of Haftenberger et al., (27). The differences were possibly due to the employment of fluoride content stated on manufacturers' label in the calculations of fluoride ingested in the German study.

In the present study, no significant correlations were found between the fluoride ingested from toothpaste or total fluoride and subsequent development of fluorosis.

Several factors may have contributed to the lack of association and include:

- questionable accuracy of retrospective recall of oral hygiene practices (28). However, methods with better evidence of reliability is yet to be available

- tendency to underestimate amount of toothpaste used. For example, Adair et al., (12) reported that parents tended to underestimate the amount of paste used in responding to selected drawings of amounts used in QA (Response indicated 0.25$0.5 \mathrm{~g}$ of dentifrice used in comparison to actual $0.689 \mathrm{~g}$ of child and $0.509 \mathrm{~g}$ of adult dentifrice actually used),

- inconsistency in amount of toothpaste used and thus the amount ingested on different occasions. Use of only one observation per patient $(3,15$, 2324 ) yields highly conservative estimates of the amount ingested chronically. Baxter (16) found subjects were not consistent in the amount ingested on different occasions (brushings). This accorded with the findings of Hargreaves et al (14), where the pattern of behaviour varies widely between children and for an individual child on different occasions. A greater betweenchild variation than within-child variation $(p<0.01)$ was however reported. Naccache et al., (21) similarly found that the difference betweenchildren was greater than within-children; the difference in the amount used between any two brushing was less than $0.25 \mathrm{~g}$ for $66 \%$ of the subjects; difference in the amount ingested was less than $0.1 \mathrm{~g}$ for $69 \%$ of the children.

- according to Barnhart (15), in the context of chronic usage, these "within patient perturbations" are zero; the variations are plusand -minus and they average out over time. 
34 Annals of Dentistry, University of Malaya, Vol. 112004

Table 4. Comparison of toothpaste usage, ingestion and fluoride exposures from swallowed toothpastes

\begin{tabular}{|c|c|c|c|c|c|}
\hline Study, age, $\mathrm{n}$ & $\begin{array}{c}\text { Amount } \\
\text { used, } \\
\text { ug }\end{array}$ & $\begin{array}{c}\text { Amount } \\
\text { ingested, } \\
\text { ug (\%) }\end{array}$ & $\begin{array}{c}\text { F dispensed } \\
\text { per } \\
\text { brushing, } \\
\text { ug }\end{array}$ & $\begin{array}{c}\text { F ingested } \\
\text { per } \\
\text { brushing, } \\
\text { ug }\end{array}$ & $\begin{array}{c}\text { F ingested / } \\
\text { day } \\
\text { ug }\end{array}$ \\
\hline
\end{tabular}

Ericson and Forsman, 1969,

$4-5, n=N A$

Hargreaves et al., 1972,

$3-6, n=44$

Barnhart et al., 1974, 2-35

Age 2-4; $n=62$

Age $5-7 ; n=56$

Glass et al., 1975;

US, 8-10; $n=67$

Baxter, 1980, London,

$5-16$ years, $n=85$

Age 5-6, $n=8$

$390-510$

$130(26.1-33.2)$

$1380 \pm 776$

$380 \pm 0.358(27.7)$

Bruun and Thylstrup 1988,

Denmark, 3-16, 179

Age 3 years, $n=63$

Age 7 years, $n=31$

$1100 \pm 680$

$1500 \pm 980$

Simard et al,1989,

Canada, 2-5, $n=23$

Age:2-3, $n=5$

$464 \pm 190$

Age 4: $n=9$

$783 \pm 280$

$651 \pm 340$

Age 5: $\mathrm{n}=9$

$622 \pm 300$

Salama et al., 1989,

$3-10$ years, $n=19$

1000 (given)

$416-545$

Naccache et al., 1990,

Quebec, 3-5, $n=48$

Naccache et al., 1992, Quebec

Age $=4 ; n=81$

Age $=5, n=77$

$446 \pm 269$

$516 \pm 366$

$130-1480 @$

$580 \pm 290$ **

NZ, 3-4 years $n=66$
$278 \pm 130(59.4)$

$390 \pm 250(48.1)$

$221 \pm 120(34.0)$

$299 \pm 190(48.1)$

$-(49.0)$

$300(34.9)$

$130(13.9)$

$117-0.123(10-12)$

$190 \pm 0.02(18.0)$

270

$360 \pm 50(36)$

$135-152(31.3-40.0)$

- (42.0)

$450 \pm 30^{*}$

$450 \pm 60^{*}$

$430 \pm 90^{*}$

$43.6 \pm 11.5$
$304 \pm 150$

$429 \pm 270$

$243 \pm 130$

$329 \pm 200$

$730 \pm 460$

Rojas-Sanchez et al., 1999,

16-40 months $\mathrm{n}=54$

Connersville, Indiana; $n=14$

Indianapolis, Indiana, $\mathrm{n}=29$

$370 \pm 80$

$\begin{array}{ll}- & 241 \pm 184 \\ - & 227 \pm 174\end{array}$

340

$0-1290$

320

(0-87\%) @

$230 \pm 230^{* *}$

Villa et al., $2000,3-5, n=20$

$273.9 \pm 175$

Haftenberger et al., 2001,

$3-6, n=11[F]=0.5 \mathrm{ppm}$

This study, proxy subjects

Index subjects

Not

Determined
$32.9 \%$

$2.9 \%$
$400.6 \pm 301.2$

"
$548 \pm 62^{*}$

$576 \pm 86^{*}$

$424 \pm 73^{*}$

$254 \pm 79$

*SEM, not SD; @ at baseline, **12 months later when age=4. 
From the standpoint of validity, it is the chronic dose of ingested dentifrice that should be used rather than the amount occasionally ingested on a random brushing. The present study had employed measurements of chronic usage within practical limits; tooth brushing being assessed over two study days, over as many as six brushing sessions for some.

- further, Spak et al., (29) had shown that bioavailability might differ between in-vitro and in-vivo situations. However, there are differences in degree of bioavailability of different fluoride compounds (higher degree of bioavailability of fluoride from NaF dentifrice $(111 \pm 13 \%, \mathrm{n}=4)$ than Na2PO3F (96 $\pm 16 \%, n=4)(8)$. Drummond and Curzon, (31) found that bioavailability of ingested fluoride can vary between $9-25 \% ; 9 \%$ for toothpaste with MFP +DCPD; 22\% for toothpaste with MFP and hydrated silica; $25 \%$ for toothpaste with MFP alone and the lower fluoride excretion from DCPD toothpaste was due to free fluoride not being as readily released from calcium abrasives.

The ready bioavailability of fluoride from toothpaste, $(7,8)$ implies that $\mathrm{F}$ dentifrices may play an important role in daily exposure of children to fluoride. The conclusion thus was ingestion of fluoride toothpaste should be minimised. Fluoride ingestion can be reduced either by limiting the amount of paste used and ingested; only a smear of paste about the size of a pea should be used, or alternatively low fluoride toothpaste should be used.

\section{CONCLUSIONS AND RECOMMENDATIONS}

It is concluded that past exposure from ingested toothpaste in 4-5 year old siblings in this study is a poor predictor of current fluorosis manifestations in 10-11 year old subjects.

Based on this study, the following research needs were identified:

1. The effectiveness of lower than conventional concentration of fluoride dentifrices for preschool children

2. Assessment of fluorosis in the younger sibling in 5 to 6 years time and the association of their current exposures with subsequent development of fluorosis.

3. This approach of determining past exposure based on proxies has not been reported elsewhere and there is a need for it to be validated.

\section{ACKNOWLEDGEMENT}

The study was conducted in response to a research need identified by the Oral Health Division, Ministry of Health, Malaysia. The first author gratefully acknowledges the scholarship from the Ministry of Science and Technology; the study leave, kind support, encouragement and permission to publish from Dato' Dr. Wan Mohamad Nasir bin Wan Othman, Director, Oral Health Division, Ministry of Health, Malaysia, as well as from Dr. Lye Munn Sann, Director, and Dr. Ng Kok Han, Head Cancer Research Centre, Institute for Medical Research, Ministry of Health, Malaysia. This study was supported by the research grants F0087/2000 and F0043/2001B from the University of Malaya.

\section{REFERENCES}

1. Dean HT, Mc Kay F. Production of mottled enamel halted by a change in common water supply. Am J Publ Health 1939; 29: 590-6.

2. Fejerskov O, Larsen MJ, Richards A, Baelum V. Dental tissue effects of fluoride. Adv Dent Res 1994; 8(1): 15-31.

3. Simard PL, Lachapelle D, Trahan L, Naccache H, Demers M, Brodeur JM. The ingestion of fluoride dentifrice by young children. J Dent Child 1989; 56: 177-81.

4. Schrotenboer G Fluoride benefits after 36 years. J Am Dent Assoc 1981; 102: 473-7.

5. Bruun C, Thylstrup A. Dentifrice usage among Danish children. J Dent Res 1988; 67(8): 1114 7.

6. Oral Health Division, Ministry of Health Malaysia. Fluoride enamel opacities in 16-yearold school children. June 2001.

7. Ellingsen JE, Ekstrand J. Plasma fluoride levels in man following intake of $\mathrm{SnF} 2$ in solution or toothpaste. J Dent Res 1985; 64(10): 1250-2.

8. Ekstrand J, Ehrnebo M. Absorption of fluoride from fluoride dentifrices. Caries Res 1980; 14 : 96-102.

9. Murakami T, Narita N, Nakagaki H, Robinson C. Fluoride intake of preschool children in Japan. Caries Res 2001; 35: 283 (Abstract no. 55). 
10. Levy SM, Kiristy MC, Waren JJ. Sources of fluoride intake in children. J Public Hlth Dent 1995; 55(1): 39-52.

11. Levy SM, Kohout FJ, Kiristy MC, Heilman JR, Wefel JS. Infants fluoride ingestion from water supplements and dentifrices. J Am Dent Assoc 1995; 126: 1625-32.

12. Adair SM, Pisciteli WP, McKnight-Hanes C. Comparison of the use of a child and an adult dentifrice by a sample of preschool children. Pediatric Dent 1997; 19(2): 99-103.

13. Amdah M. Fluoride toothpaste usage among preschool children. University of Malaya. MCD Thesis 2000.

14. Hargreaves JA, Ingram GS, Wagg BJ. A gravimetric study of the ingestion of toothpaste by children. Caries Res 1972; 6: 237-43.

15. Barnhart WE, Hiller LK, Leonard GJ, Michaels SE. Dentifrice usage and ingestion among four age groups. J Dent Res 1974; 53(6): 1317-22.

16. Baxter PM. Toothpaste ingestion during tooth brushing by school children. Br Dent J 1980; 148(5): 125-8.

17. Dowell TB. The use of toothpaste in infancy. $\mathrm{Br}$ Dent J 1981; 150: 247-9.

18. Dean HT. Classification of mottled enamel diagnosis. J Am Dent Assoc 1934; 21: 1421-6.

19. Dean HT. The investigation of physiological effects by the epidemiological method. In: Moulton FR, editor. Fluoride and dental health. Pub No. 19 Washington DC. American Association for the advancement of Science 1942, 23-31.

20. World Health Organisation. Health Research Methodology. A guide for training in research method. WHO Regional Publications-Western Pacific Education in action series no.5. Manila: WHO 1992.

21. Naccache H, Simard PL, Trahan L, Demers M, Lapointe C, Brodeur JM. Variability in the ingestion of toothpaste by preschool children. Caries Res 1990; 24: 359-63.
22. Ericsson Y, Forsman B. Fluoride retained from mouth rinses and dentifrices in pre-school children. Cries Res 1969; 3(3): 290-9.

23. Salama F, Whitford GM, Barenie JJ. Fluoride retention by children from tooth brushing. J Dent Res 1989; 68(spec issue): 335 (Abstract no:1227)

24. Naccache H, Simard PL, Trahan L, Brodeur JM, Demers M, Lachapelle D, Bernard PM. Factors affecting the ingestion of fluoride dentifrice by children. J Public Health Dentistry 1992: 52(4): 222-6.

25. Rojas-Sanchez F, Kelly SA, Drake KM, Eckert GJ, Stookey GK, Dunipace AJ. Fluoride intake from foods, beverages and dentifrices by young children in communities with negligibly and optimally fluoridated water: a pilot study. Community Dent Oral Epidemiol 1999; 27: 288-97.

26. Villa AE Anabalon M, Cabezes L. The fractional urinary fluoride excretion in young children under stable fluoride intake conditions. Community Dent Oral Epidemiol 2000; 28: 344-55.

27. Haftenberger M, Viergutz G, Neumeister V, Hetzer G. Total fluoride intake and urinary excretion in German children aged 3-6 years. Caries Res 2001; 35: 451-7.

28. Kwan SYL, Williams SA. The reliability of interview data for age at which infants' tooth cleaning begins. Community Dent Oral Epidemiol 1998; 26: 214-8.

29. Spak CJ, Ekstrand J, Zylberstein D. Bioavalability of fluoride added to baby formula and milk. Caries Res 1982; 16: 249-56.

30. Drummond BK, Curzon MES. Urinary excretion of fluoride following ingestion of MFP toothpastes by infants aged two to six years. J Dent Res 1985; 64(9): 1145-8. 